\title{
Physicochemical Properties and Control Release of Aloe Vera (Aloe barbadensis Miller) Bioactive Loaded Poly (Lactic Co-Glycolide Acid) Synthesized Nanoparticles
}

\author{
Lamin S. Kassama*, Jawadul Misir
}

Food Engineering Laboratory, Department of Food and Animal Sciences, A-101 Carver Complex Thomas Wing South, Alabama A\&M University, Normal, AL, USA

Email: *Lamin.kassama@aamu.edu

How to cite this paper: Kassama, L.S. and Misir, J. (2017) Physicochemical Properties and Control Release of Aloe Vera (Aloe barbadensis Miller) Bioactive Loaded Poly (Lactic Co-Glycolide Acid) Synthesized Nanoparticles. Advances in Chemical Engineering and Science, 7, 333-348.

https://doi.org/10.4236/aces.2017.74025

Received: June 29, 2017

Accepted: August 12, 2017

Published: August 15, 2017

Copyright $\odot 2017$ by authors and Scientific Research Publishing Inc. This work is licensed under the Creative Commons Attribution International License (CC BY 4.0).

http://creativecommons.org/licenses/by/4.0/

c) (i) Open Access

\begin{abstract}
Nano-encapsulation is a platform which offers a promising application for control release and the delivery of drugs in pharmaceuticals and antioxidant/antimicrobial in food systems. Poly (lactic-co-glycolide acid) (PLGA) is a biodegradable and biocompatible co-polymer of lactic acid and glycolic acid which is used for synthesizing food based polymeric nanoparticles (NP). The aim of this study was to evaluate the morphological and physicochemical properties and the controlled release of bioactive components derived from Aloe vera gel loaded PLGA NP. The results shows the mean hydrodynamic diameter of the unloaded NP is $103 \mathrm{~nm}$ which is significantly $(p<0.01)$ smaller than the loaded freeze dried powered gel (FDG) $(147 \mathrm{~nm})$ and liquid gel (LG) $(221 \mathrm{~nm})$ and the particle size distribution given by the Poly-dispersity Index were 0.2, 0.2 and 0.3 , respectively. The zeta potential for unloaded, FDG and LG NP were $\pm 60, \pm 28$ and $\pm 22 \mathrm{mV}$, respectively, hence were electrokinetically stable NP. No significant $(p>0.05)$ inhibition of the antioxidant potential was observed with loaded NP. The entrapment efficiency for the FDG synthesized was $87 \%$, and the burst effect was observed after $4 \mathrm{~h}$ as a result of the encapsulation effect. The release kinetics of bioactive is govern by the combination of mass diffusion and capillary action.
\end{abstract}

\section{Keywords}

Nanoencapsulation, PLGA, Aloe Vera Gel, Size Distribution, Time Release Kinetics \& Antioxidant 


\section{Introduction}

The importance of reactive oxygen species (ROS) and free radicals has attracted increasing attention over the past decade due to their impact on human health. The ROS include free radicals such as superoxide anion radicals $\left(\mathrm{O}_{2}^{-}\right.$. ), hydroxyl radicals $\left(\mathrm{OH}^{-}\right)$and non-free radical species such as $\mathrm{H}_{2} \mathrm{O}_{2}$ and singlet oxygen $\left(\mathrm{O}_{2}\right)$, these are various forms of activated oxygen. They are continuously produced during normal physiological process and they can easily initiate the per-oxidation of membrane lipids, causing oxidative stress leading to diseases like diabetes mellitus, cancer and cardiovascular diseases [1] [2] [3]. It accelerates deterioration and development of rancidity in food systems containing lipid, hence the production of the off flavor compounds, polymerization and reducing the nutritive value of the food products [4] [5].

Renolds \& Dweck [6] has reported that phenolic compounds have the ability to trap the free radicals or scavenge them through a series of reactions with enzymes. Hence, many research efforts are underway in developing novel methods of introducing natural antioxidants from fruits, vegetables, herbs and spices in the food system. Aloe vera (Aloe barbadensis Miller) a member of the family $\mathrm{Li}$ liaceae, is a short stemmed succulent, perennial herb. The gel found in the Aloe vera leaves is rich with bioactive compounds such as: vitamins, minerals, amino acids, enzymes, sugars, hormones and antioxidants. The efficacy of the in vitro antioxidant activities of the gel extracts shows the potential for reducing lipid peroxidation/oxidative stress with consequential health benefits [7].

The bioaccessibility of these bioactive compounds has been limited due to the harsh conditions encountered during food processing. Processing parameters such as temperature, light, $\mathrm{pH}$ severely degrade these bioactive components, furthermore conditions ( $\mathrm{pH}$, enzymes) in the gastrointestinal tracts or during storage when exposed to light and oxygen [8] [9]. However, Barras et al. [10] \& Weiss et al. [11] believe that developing a control delivery mechanism of bioactive components via nanoencapsulation will provide protection from the harsh environmental conditions. Azimi et al. [12] \& Freiberg \& Zhu [13] alluded that the particle size and morphology of the NP are important properties that influence the release of the active compounds.

A mean Poly (Lactic co-Glycolide Acid) (PLGA) NP diameter (148 nm) loaded with (antioxidant thermoquinone) NP was reported by Nallamuthu, Parthasarathi, \& Khanum [14] and the measure of the colloidal dispersal and uniformity was characterized based on the polydispersity index (PDI) (0.1). The PSD is influenced by the electrostatic repulsion between particles disperse in the liquid suspension, hence there is a strong correlation between the electrokinetic potential and PDI. A PDI value less than 0.1 indicates a homogenous and mono-dispersity and hence a better particle size distribution [15], and Qi et al. [16] reported a zeta potential values of $\pm 30 \mathrm{mV}$ as a standard value for a stable nanoemulsion with less tendency for particle aggregation.

The particle size, morphology and zeta potentials of loaded NP are significant 
properties that control the entrapment efficiency (EE) and the time releases of the bioactive compound. Nanoparticles synthesize with polymers such as Poly (Lactic co-Glycolide Acid) were reported to have a high EE due to its molecular structure that is associated to its active sites [17]. An EE of $47.6 \%$ and $38.9 \%$ for cinnamon bark extract loaded in PLGA-50 and PLGA-65 NP, respectively was reported by Hill et al. [18]. Peng et al. [19] also reported about $80 \%$ time release of plant oil curcumin encapsulated with PF127 micelles during 6-h period at $37^{\circ} \mathrm{C}$. The control release of active compounds is govern by Fick's law of diffusion, when a species of bioactive entrapped at specific site (initial position) migrates through a polymeric a matrix to the surface and subsequent release into the medium surrounding the NP [20], hence influenced the concentration differences combined with electrokinectic and other physical properties. Therefore, the aim of this study was to evaluate the physicochemical properties and the controlled release of bioactive components derived from Aloe vera gel loaded PLGA NP.

\section{Materials and Method}

\subsection{Sample Preparation}

Poly (lactic-co-glycolide) acid (1:1, MW: 10,000 - 15,000 Da), DMAB (Dimethylamine borane), ethyl acetate and HPLC grade water were purchased from Fisher Scientific. Aloe vera gel (100\%) was collected from the Aloe vera plant grown at the Alabama A \& M University's greenhouse.

\subsection{Freeze Drying of Aloe Vera Gel}

The harvested leaves were properly washed with portable water and sliced open to extract the gel using an ethanol sterilized surgical knife. The extract was blended using a coffee grinder and pour into a freeze drying glass canisters. The gel samples in the freeze frying glass canisters were frozen overnight at $-20^{\circ} \mathrm{C}$. The frozen samples were freeze dried using a freeze dryer (Labconco FreeZone-6, Kansas City, Mo) at $-52^{\circ} \mathrm{C}$ and 0.808 mbar for $48 \mathrm{~h}$.

\subsection{Synthesis of PLGA Loaded Nanoparticle}

Nanoparticles were formed using the ultra-sonication solvent evaporation technique. The organic phase was formulated by dissolving PLGA in ethyl acetate along with about $44 \mathrm{mg}$ of the samples (freeze dried Aloe veragel and liquid Aloe vera gel) at a ratio of 1:9 relative to PLGA. The samples were vortexed to dissolve the PLGA. The organic phase was added drop-wise to the aqueous $0.5 \%(\mathrm{w} / \mathrm{v})$ DMAB solution with water (HPLC grade) and continually stirring with a Magnetic stirrer. Once all the compound/polymer mixture was added the emulsion was sonicated (Sonicator 3000, Ultrasonic liquid processor, Church Hill, CT) at $75 \mathrm{~W}$ for $15 \mathrm{~min}$. Then the organic solvent, ethyl acetate, present in the emulsion was evaporated using Rotary vacuum evaporator (RE 301, Yamato Scientific Co. LTD, Tokyo, Japan) at $40^{\circ} \mathrm{C}$. The same procedure was repeated for synthesizing 
the unloaded (control) without the gel. The NP were collected by centrifugation at $10,000 \mathrm{~g}$ for $20 \mathrm{~min}$ at $4^{\circ} \mathrm{C}$. Finally, recovered NP was resuspended in $2 \mathrm{~mL}$ cryoprotectent solution ( $2 \%$ sucrose) and freeze dried using the same methods as described above.

\section{Physicochemical Characterization of the Loaded Nanoparticles}

\subsection{Particle Size Distribution and Polydispersity Index}

The freeze dried NP was suspended in distilled water at the concentration of 10 $\mathrm{mg} / \mathrm{mL}$ and $2 \mathrm{~mL}$ of each sample was pipetted into a quartz cuvette and the particle size distribution (PSD) and the polydispersity index (PDI) were measured using the dynamic laser scattering Malvern Zetasizer Nano series (Nano ZS90, Malvern Instruments Ltd., Worcestershire, UK) according to the method developed by Kassama et al. [21]. The instrument was pre-programmed to take three consecutive readings by intensity and volume and each sample was measured in triplicate.

\subsection{Electrokinetic Potential and Surface Charge}

About $1 \mathrm{~mL}$ of each sample was pipetted into a zeta potential capillary cell which was placed in the Dynamic Light Scattering Malvern Zetasizer Nano series (Nano ZS90, Malvern Instruments Ltd., Worcestershire, UK) according to the method developed by Kassama et al. [21]. The electrokinetic potentials (Zeta potential) of the NP was measured, hence the values obtained were used to determine the stability and the materials tendency to aggregate. The instrument was preprogrammed to take three consecutive readings by intensity and volume and each sample was measured in triplicate.

\subsection{Structural Morphology of the PLGA Nanoparticles}

The morphology of the nanoparticle was determined by a transmission electron microscope (TEM) at University of Auburn, Auburn, AL at an accelerating voltage of $60 \mathrm{kV}$. About $10 \mu \mathrm{L}$ of aqueous suspension of particles were placed on 300 mesh copper grids and stained with a $2 \%(\mathrm{w} / \mathrm{v})$ phosphotungstic acid in $\mathrm{dH}_{2} \mathrm{O}$ to provide a contrast under magnification. The suspension was allowed to dry before viewing at 40,000 to $100,000 \times$ magnification.

\subsection{Entrapment Efficiency of PLGA Loaded PLGA NP}

The entrapment efficiency was determined as a function of the decreased DPPH concentration over time. The NP were dispersed in the $95 \%$ acetonitrile solution for a period of $72 \mathrm{~h}$ with periodic mixing to allow bioactive compounds to be diffused in the solution. The solutions were centrifuged at $3000 \mathrm{~g}$ to separate from PLGA. About $1 \mathrm{~mL}$ of the supernatant collected was mixed with $3 \mathrm{~mL}$ of $0.1 \mathrm{mM}$ DPPH solution. The $95 \%$ acetonitrile gel solution $(1 \mathrm{~mL})$ was taken as the control. The reaction kinetics of the DPPH solution recovered from NP and 
control gel solutions determined by using the microplate spectrophotometer (Spectra-Max 250, Molecular Device Corp., Sunnyvale, CA) at $517 \mathrm{~nm}$ for 24 hours. The same procedure was repeated for the Aloe vera liquid samples. The entrapment efficiency was calculated as shown in Equation (1):

$$
\mathrm{EE}(\%)=\frac{\text { The reaction time of the samples }}{\text { The reaction time of the control }} \times 100
$$

\subsection{Control Release Kinetics of Loaded PLGA Nanoparticles}

Release kinetics methodology was developed based on the modified version of the DPPH antioxidant potential method developed by Braca et al. [22]. About 1 $\mathrm{mg} / \mathrm{mL}$ concentration of the freeze dried aloe vera gel Loaded PLGA NP was dropped in $3 \mathrm{~mL}$ of $0.1 \mathrm{mM}$ DPPH solution. The reduction of the DPPH concentration was observed over time relative to the freeze dried Aloe vera gel of the same concentration. The absorbance was measured with microplate spectrophotometer (Spectra-Max 250, Molecular Device Corp., Sunnyvale, CA) at 517 $\mathrm{nm}$ as the DPPH concentration decreases. The release kinetic was estimated based on the decrease in DPPH which is inversely proportional to the release of the bioactive components from loaded NP that reacts with the DPPH solution. Hence, the rate constant was determined by applying following Equation (2):

$$
\ln \left(\frac{A-A_{0}}{A_{0}-A_{\alpha}}\right)=K t
$$

where $A$ is the absorbance at time $t, A_{0}$ is the absorbance at time $0, A_{\alpha}$ is the final absorbance and $K$ is the release rate constant of the bioactive component released in the medium.

\subsection{Antioxidant Activity of the Encapsulated Loaded PLGA NP}

The modified 2,2-diphenyl-1-picrylhydrazyl(DPPH) assay used in this study was based on the method proposed by Nallamuthu et al. [14] \& Braca et al. [22]. About $1 \mathrm{~mL}$ of the aliquots of different concentrations $(0.1-1 \mathrm{mg} / \mathrm{mL})$ of the methanolic solutions containing different levels of the samples treatments were added to $3 \mathrm{~mL}$ of $0.1 \mathrm{mM}$ DPPH solution. The mixtures were agitated and kept at room temperature for 45 minutes. Freeze dried Aloe vera gel $(0.1-1 \mathrm{mg} / \mathrm{mL})$ in methanolic solution was used as the control. The decrease in the solution absorbance due to proton donating activity of bioactive components of the NP was determined with a microplate spectrophotometer (Spectra-Max 250, Molecular Device Corp., Sunnyvale, CA) at $517 \mathrm{~nm}$. Absorbance reading was taken at every $30 \mathrm{~min}$ interval for $90 \mathrm{~min}$. Lower absorbance of the reaction mixture indicated higher free radical scavenging activity. The DPPH radical scavenging activity was calculated using the formula shown by Equation (3):

$$
\text { DPPH Radical Scavenging Activity }(\%)=\left[\frac{A^{0}-A^{1}}{A^{0}}\right] \times 100
$$

where $A^{0}$ is the absorbance of the control, and $A^{1}$ is the absorbance of treatment 
or standard sample.

\section{Results and Discussion}

\subsection{Size, Particle Size Distribution and Poly Dispersity Index of Aloe Vera Gel Loaded NP}

The freeze dried and liquid Aloe vera gel were used with PLGA to synthesize Aloe vera freeze-dried powder (FDG) and liquid-gel (LG) loaded NP. The results of the physicochemical measurements of the synthesized FDG and LG loaded NP are shown in Table 1. The particle size, morphology, surface charge and physical state are the critical factors that influence the functional performance of the NP [23]. The results of the study shows the state of the materials either synthesized in the dry powdered or liquid form of NP significantly $(p<0.05)$ influence the physicochemical and morphology of the NP. The mean hydrodynamic diameter of the unloaded PLGA NP was $103 \mathrm{~nm}$ which is significantly $(p<0.01)$ smaller than the loaded FDG (147 nm) and LG $(221 \mathrm{~nm})$, a similar trend was observed for the actual diameters measured with TEM and recorded values of 60, 100 and $200 \mathrm{~nm}$, respectively, is shown in Table 1. The larger particles sizes for the LG NP is largely associated to its surface tension, which in effect limit its dispersity in the nanoemulsion [24], hence the results were also similar to the studies conducted with plant extracts and drugs. Bhattacharyya et al. [25] also reported a mean hydrodynamic diameter of $123 \mathrm{~nm}$ for PLGA encapsulated ethanolic extract and Pereira et al. [26] reported a size range of 145 to $162 \mathrm{~nm}$ for the nanoencapsulated hydrophobic phytochemical with PLGA.

No significant differences $(p>0.05)$ of the materials synthesized either in the PLGA unloaded NP and FDG loaded NP was observed on the PDI values of 0.2 (Table 1), a similar observation was also reported by Hill et al. [18] for a cinnamon bark extract encapsulated with PLGA NP. The LG loaded NP shows a less homogeneity compared to the FD loaded NP with a PDI of 0.3 as shown in Table 1, this was also manifested in the broadening of the PSD bell-shape-curved as shown in Figure 1(c).

Table 1. The physicochemical properties and the entrapment efficiency (EE) of Aloe vera gel loaded gel Poly (lactic co-Glycolide Acid) Nanoparticles.

\begin{tabular}{cccccc}
\hline Treatments & $\begin{array}{c}\text { Hydrodynamic } \\
\text { Diameter } \\
(\mathrm{nm})\end{array}$ & $\begin{array}{c}\text { TEM } \\
\text { Diameter } \\
(\mathrm{nm})\end{array}$ & $\begin{array}{c}\text { Zeta Potential } \\
(\mathrm{mV})\end{array}$ & $\begin{array}{c}\text { Polydispersity } \\
\text { index }\end{array}$ & $\begin{array}{c}\text { EE } \\
(\%)\end{array}$ \\
\hline PLGA Unloaded NP & $102.9 \pm 0.35^{\mathrm{a}}$ & 60 & -60 & 0.2 & - \\
$\quad \begin{array}{c}\text { Freeze Dried } \\
\text { Aloe Vera gel } \\
\text { Loaded PLGA NP }\end{array}$ & $146.2 \pm 1.06^{\mathrm{b}}$ & 100 & -28 & 0.2 & 86.3 \\
$\begin{array}{c}\text { Liquid Aloe } \\
\text { Vera gel }\end{array}$ & $221.4 \pm 0.86^{\mathrm{c}}$ & 200 & -21.9 & 0.3 & 67.0 \\
Loaded PLGA NP & & & & & \\
\hline
\end{tabular}




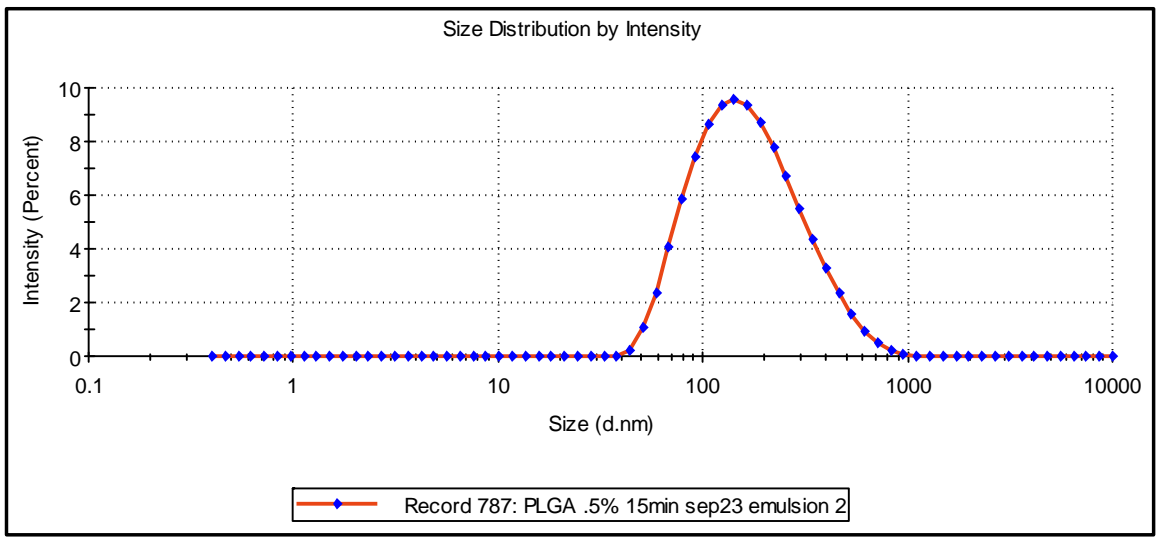

(a)

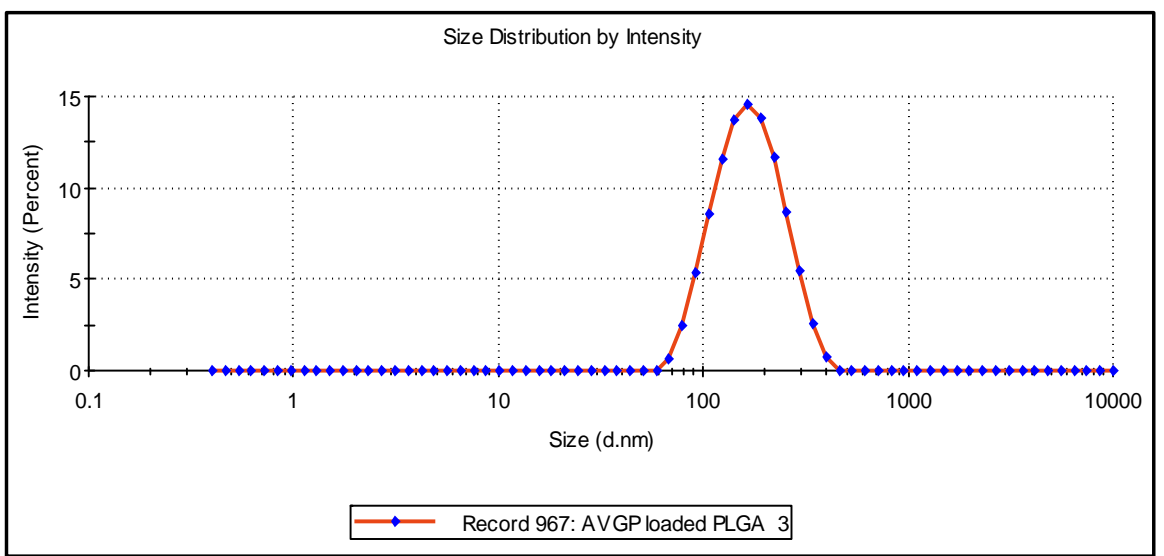

(b)

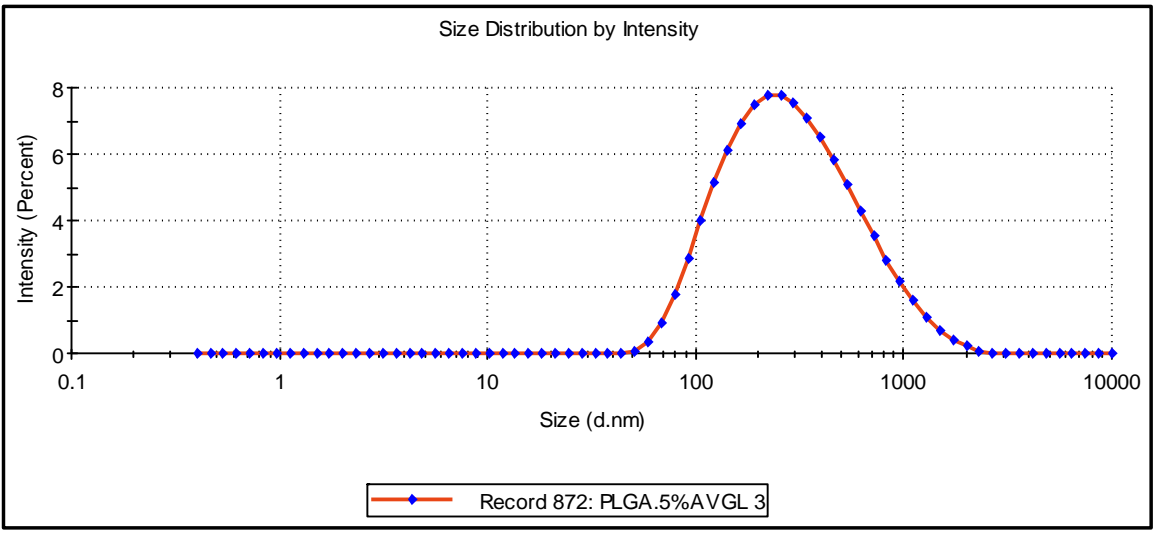

(c)

Figure 1. The DLS characterization of the particles size distribution for Aloe vera gel loaded NP: (a) PLGA unloaded (no Aloe vera gel) NP, (b) FDG with PLGA loaded NP, (c) LG with PLGA loaded NP.

\subsection{Electrokinetic Potential and Surface Charges}

The NP dispersed in solution are usually energetic and under constant motion, and the repulsive forces between adjacent charges particles is one of the determinant factors to particle stability. Hence, the electrokinetic potential is a measure of the charges to establish stability of the NP. The results of the colloidal 
dispersion measured for this study varies within $\pm 60 \mathrm{mV}$ as shown in Figures $2(a)-(c)$, hence significantly different $(p<0.05)$ amongst the different treatments.

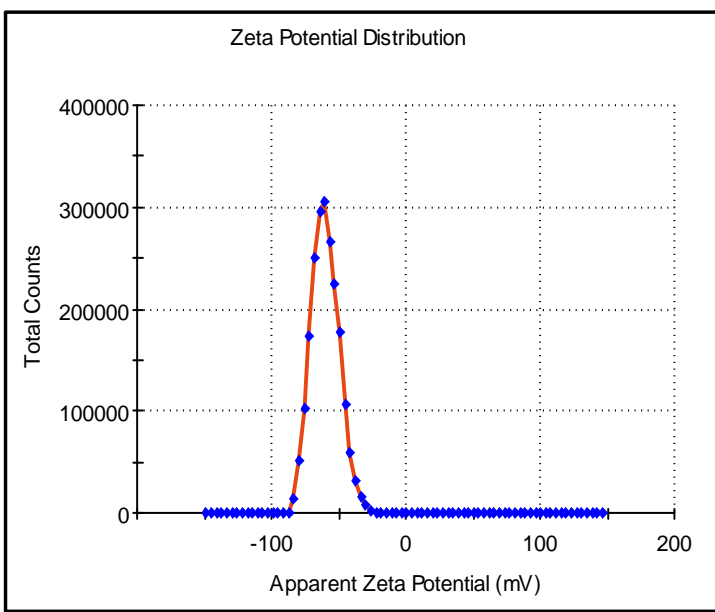

(a)

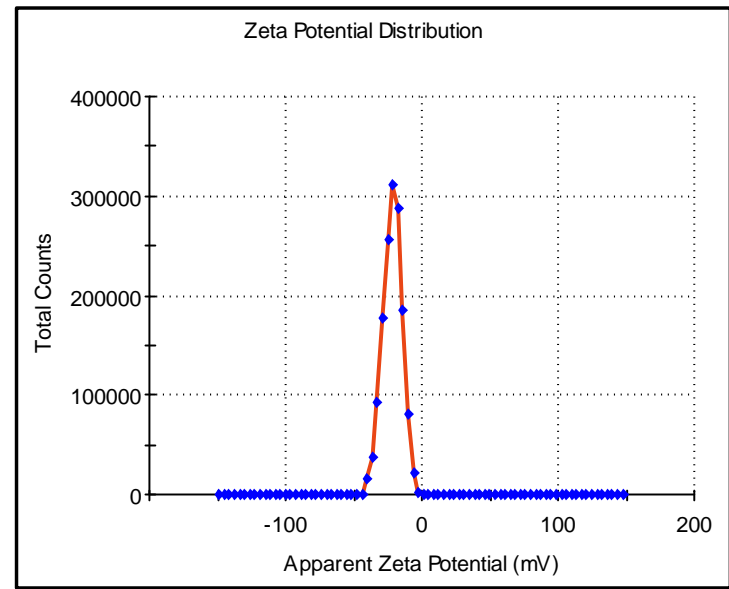

(b)

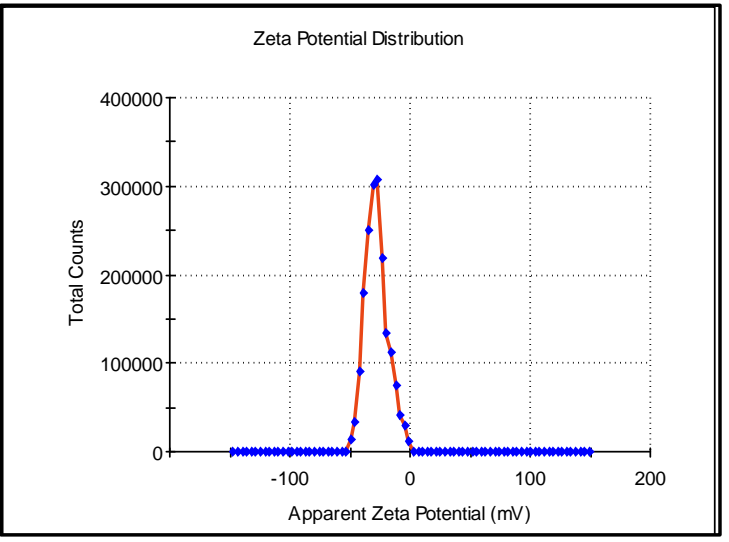

(c)

Figure 2. The DLS characterization of the electrokinetic potential and surfacecharges for Aloe vera gel loaded NP: (a) PLGA unloaded (no Aloe vera gel) NP, (b) FDG with PLGA loaded NP, (c) LG with PLGA loaded NP. 
The mean zeta potential values were $-60 \mathrm{mV},-28 \mathrm{mV} \&-21 \mathrm{mV}$ for the unloaded, FDG and LG NP, respectively as shown in Figures 2(a)-(c). The zeta potential value for unloaded NP were higher than the loaded NP, this is largely due the influence of the bioactive compounds attached to the polymer charge, hence the adsorption of the bioactive compounds on the surface of the nano-carriers. The zeta potential values reported in this study shows strong electrostatic repulsive forces, hence will prevent particle aggregation, which ultimately suggests a highly stable emulsion. Pool et al. [27] reported similar observations for unloaded, quercetin loaded and catechin loaded nanoparticles as \pm 31 , \pm 45 and $\pm 30 \mathrm{mV}$, respectively.

\subsection{Particle Size, Structure and Morphology}

The size and structural morphology of the unloaded, FDG and LGNP are important characteristic features in the control release of encapsulated compound, hence the image by TEM was carried to determine their formation as shown in Figures 3(a)-(c). Figure 3 also shows varieties of particles of different sizes were

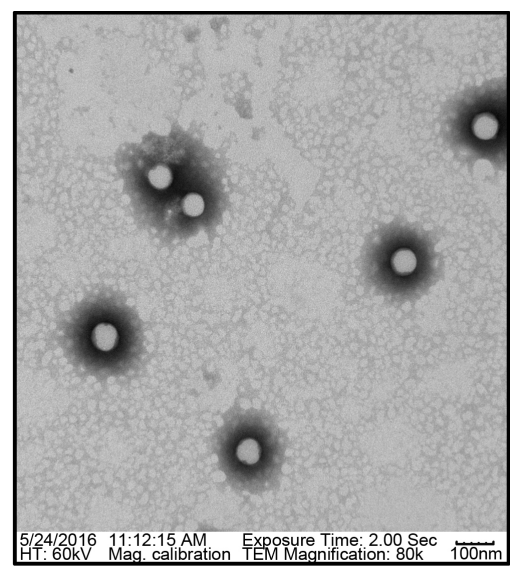

(a)

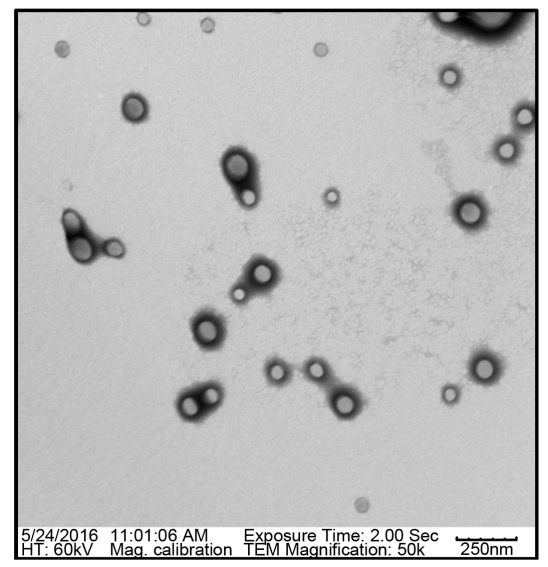

(b)

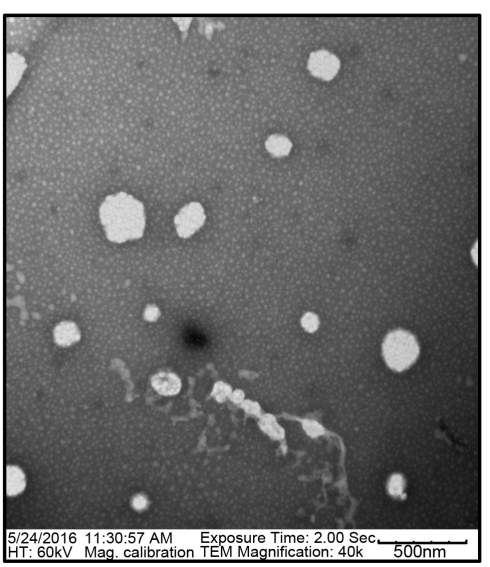

(c)

Figure 3. The TEM micrograph characterization of the particle size, structure and morphology for Aloe vera gel loaded NP: (a) PLGA unloaded (no Aloe vera gel) NP, (b) FDG with PLGA loaded NP, (c) LG with PLGA loaded NP. 
present in the nano-emulsion. The actual particle diameters measures with TEM are shown in Table 1, in comparison, Hill et al. [18] reported 167, 162, 152, 145 $\mathrm{nm}$ for PLGA50-CBE, unloaded PLGA50, PLGA65-CBE and unloaded PLGA65, respectively. The structural morphology of unloaded PLGA, FDG, and LG loaded NP are sphericalin shape and slightly polydisperse $(0.2,0.2$, and 0.3$)$, respectively. The dark segment around the periphery of the NP is the manifestation of the coroner effect as shown in Figure 3(a) and Figure 3(b), which could be attributed to the DMAB and or the phosphotungstic acid in $\mathrm{dH}_{2} \mathrm{Othat}$ may have formed between the hydrophobic region and the PLGA chain, while the hydrophilic regions of DMAB [15] [18]. However, in the case of LG loaded NP a rough surface could be seen as shown in Figure 3(c), this could be attributed to the gel adsorption to the periphery of the NP.

\subsection{Entrapment Efficiency}

The entrapment efficiency (EE) was determined based on the method described above and the values were calculated using Equation (1). The EE of the FDG synthesized is significantly different $(p<0.05)$ from the LG loaded NP, hence the values determined were $87 \%$ and $67 \%$, respectively. These values are much higher than $48 \%$ and 39\% for PLGA50-CBE and PLGA65-CBE, receptively reported by Hill et al. [18]. The EE values for the FDG loaded NP was much higher due to the increased concentration of the bioactive compounds due to freeze drying. Alam et al. [28] reported 63\% for TQ-chitosin NP whereas $90 \%$ was reported for TQ-liposome NP [29]. Many factors influences the control and regulation of the EE such as the: entrapped material, polymer molecular weight, and lactide: glycolide ratio [30] [31] [32], likewise the interaction between the hydroxyl group of the active compound and carboxyl group of the PLGA.

\subsection{Controlled Time Release Kinetics}

The controlled release kinetic profile of bioactive component derived from Aloe vera gel encapsulated with PLGA was studied as a function of decrease bioactive as shown in Figure 4(a) and Figure 4(b) for Aloe vera gel and FDG PLGA NP. The association of the mas transfer process to the release of the bioactive from encapsulated matrix into the aqueous solution is govern by the combination of diffusion and capillary action. The mass transfer process occurs in three distinct stages, hence categorized into phases of mass transfer as observed during the period of $10 \mathrm{~h}$ for the FDG loaded NP as shown in Figure 4(b). The first phase (Ph-I) exhibited a rate constant of $1.2 \times 10^{-3} \mathrm{~min}^{-1}$ during the first $4 \mathrm{~h}$ of mass transfer and is five-fold much slower than the rate $\left(6.5 \times 10^{-3} \mathrm{~min}^{-1}\right)$ observed during the second phase (Ph-II) as shown in Table 2. The factors that contributed to the slow rate of the first phase could be attributed to the protective effect of the nano-coatings. More importantly, the integrity of the encapsulating polymer to degrade in the medium, the affinity of the active compound to the PLGA, matrix porosity, polymer erosion and the polymer swell and degradation 


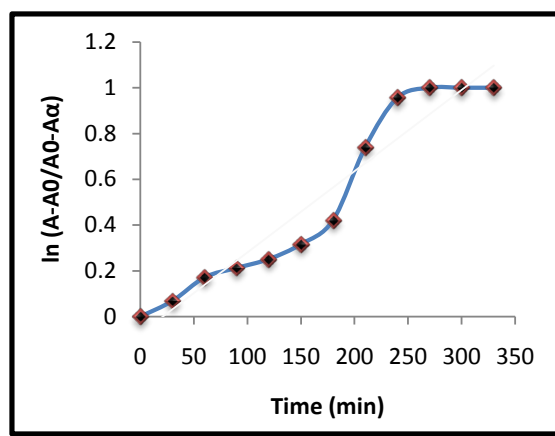

(a)

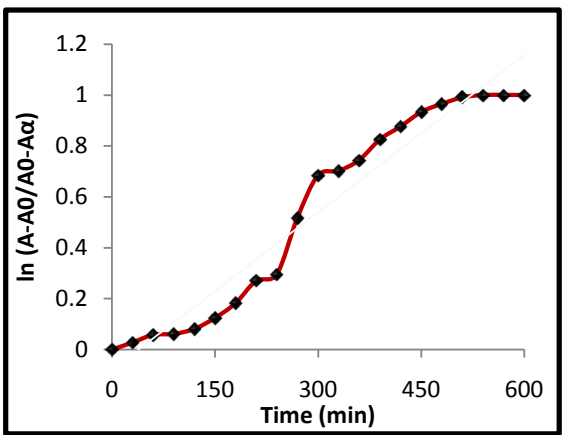

(b)

Figure 4. Controlled release of encapsulated bioactive compound derived from Aloe vera gel with PLGA into: (a) Freeze dried Aloe vera gel (no PLGA included), (b) Aloe vera FDG loaded NP with PLGA.

Table 2. Controlled release mass transfer rate constant of the Aloe vera bioactive components migration from the loaded PLGA NP to the surrounding DPPH medium.

\begin{tabular}{ccccc}
\hline \multirow{2}{*}{$\begin{array}{c}\text { Time } \\
(\mathrm{min})\end{array}$} & \multicolumn{2}{c}{ Freeze dried Aloe vera gel } & \multicolumn{2}{c}{$\begin{array}{c}\text { Freeze dried Aloe vera gel } \\
\text { Loaded PLGA NP }\end{array}$} \\
\cline { 2 - 5 } & Rate Constant $\left(\mathrm{min}^{-1}\right)$ & $\mathrm{R}^{2}$ & Rate Constant $\left(\mathrm{min}^{-1}\right)$ & $\mathrm{R}^{2}$ \\
\hline Ph-I: $0-240$ & $3.6 \times 10^{-3}$ & 0.88 & $1.2 \times 10^{-3}$ & 0.93 \\
Ph-II: $240-300$ & - & - & $6.5 \times 10^{-3}$ & 0.99 \\
Ph-III: $300-540$ & - & - & $1.5 \times 10^{-3}$ & 0.97 \\
\hline
\end{tabular}

leading to burst effect [5] [18] [33].The burst effect is a phenomenon discussed by Hill et al. [18] observed during the first-hour of their study with Cinnamon back extract with PLGA NP, in this study, the effect was observed to have occurred during Ph-II of the mass transfer after $4 \mathrm{~h}$. Hence, the movement of mass during this phase is the combination of diffusion and capillary action. The mass diffusion of the bioactive slows down during the third-phase (Ph-III) to $1.5 \times$ $10^{-3} \mathrm{~min}^{-1}$ after $5 \mathrm{~h}$ and eventually plateaus after 9 to $10 \mathrm{~h}$ period as shown in Figure 4(b) due to concentration differences between the NP and the surrounding medium. In contrast, the rate constant $\left(3.6 \times 10^{-3} \mathrm{~min}^{-1}\right)$ for the mass transfer of the bioactive from the freeze dried Aloe vera gel (Figure 4(a)) was 3 times faster than in the FDG loaded NP during the Ph-I periods $(4 \mathrm{~h})$ as shown in Table 2. Hence, the delayed of PLGA degradation is manifested by impeding the mass transfer of the bioactive and provide a longer release time. The rate constant model (Equation (2)) fits well with the experimental data for gel loaded NP (Table 2).

\subsection{Antioxidant Activity of the Encapsulated Lyophilized Aloe Vera Gel}

The 2,2-diphenyl-1-picrylhydrazyl (DPPH) assay was used to determine the effect of the antioxidant potentials on nanoencapsulation of PLGA NP, hence the inhibition profile is shown in Figure 5(a) and Figure 5(b). The principle of the 


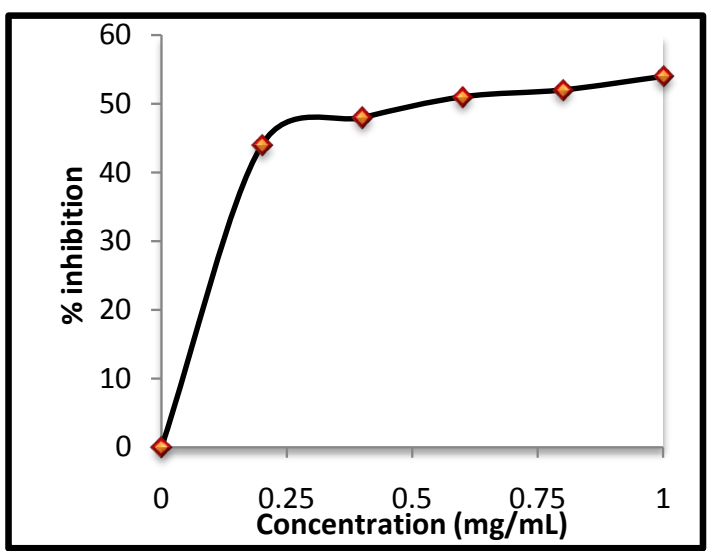

(a)

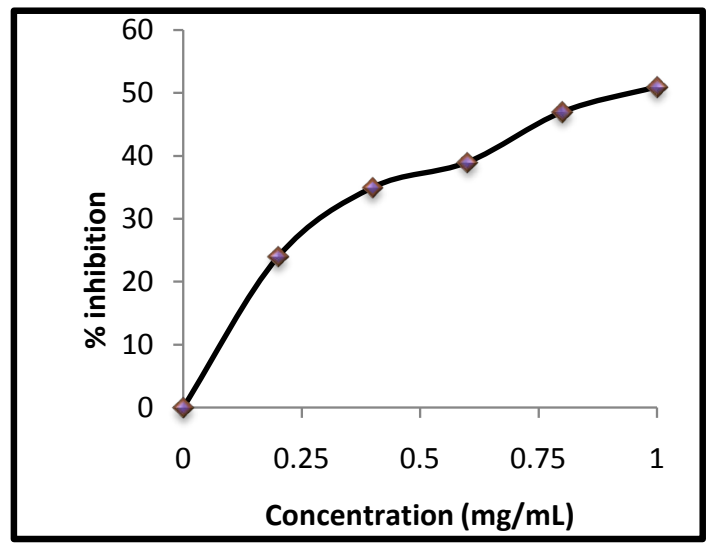

(b)

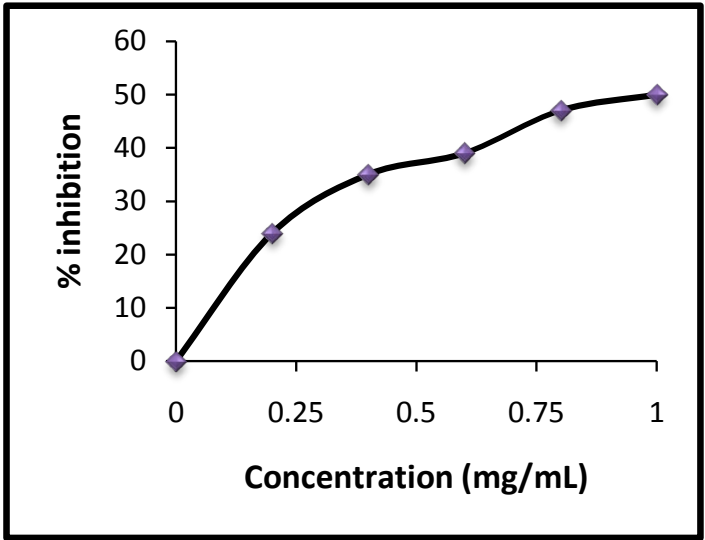

(c)

Figure 5. DPPH radical scavenging antioxidant potentials of encapsulated bioactive compound derived from Aloe vera gel with PLGA into: (a) Freeze dried Aloe vera gel (no PLGA included), (b) Aloe vera FDG loaded NP with PLGA, (c) Aloe vera LG loaded NP with PLGA.

assay was based on the reduction of hydrogen donating antioxidant due to the formation of diphenyl-1-picrylhydrazyl. The DPPH free radical scavenging 
power for the FDG loaded PLGA NP was 51\% as shown in Figure 5(c), and 54\% inhibition for freeze dried Aloe vera gel (Figure 5(b)), and 50\% for the LG loaded NP with PLGA (Figure 5(a)). Hence, no significant difference $(p>0.05)$ exist between the three, therefore the encapsulation in the PLGA has not negatively impacted the antioxidant potentials of the Aloe vera bioactive. Mathew et al. [34] made a similar observation on the antioxidant potential for the curcumin loaded PLGA NP.

\section{Conclusion}

Freeze dried powdered Aloe vera gel and Aloe vera gel liquid nanoparticles were synthesized with PLGA by using the solvent evaporation technique. The results by all indications show significant effect $(p<0.05)$ of Nano-encapsulation on the physicochemical properties of the nanoparticles. The mean hydrodynamic diameter of the unloaded PLGA NP is $103 \mathrm{~nm}$ which is significantly $(p<0.05)$ smaller than the loaded Aloe vera FDG $(147 \mathrm{~nm})$ and LG $(221 \mathrm{~nm})$ and the measured electrokinetic values indicates stable nanoparticle. No significant $(p>$ 0.05 ) inhibition of the antioxidant potential was observed with encapsulated NP. The entrapment efficiency for the FDG synthesized was $87 \%$, hence the burst effect was observed after $4 \mathrm{~h}$ as a result of the encapsulation effect. The release kinetics of bioactive is govern by the combination of mass diffusion and capillary action.

\section{Acknowledgements}

The authors' wishes to acknowledge the financial support of the USDA National Institute of Food and Agriculture, [USDA-NIFA Capacity Building Grant Project Title: Nanotechnology Application in the Food Engineering Curriculum. Accession number 230755]. Likewise the AAMU experimental station for committing resources in support of the research project.

\section{References}

[1] Seki, S., Kitada, T., Yamada, T., Sakaguchi, H., Nakatani, K. and Wakasa, K. (2002) In Situ Detection of Lipid Peroxidation and Oxidative DNA Damage in Non-Alcoholic Fatty Liver Diseases. Journal of Hepatology, 37, 56-62. https://doi.org/10.1016/S0168-8278(02)00073-9

[2] Baynes, J.W. and Thorpe, S.R. (1999) Role of Oxidative Stress in Diabetic Complications: A New Perspective on an Old Paradigm. Diabetes, 48, 1-9. https://doi.org/10.2337/diabetes.48.1.1

[3] Gross, M.D. (2008) Lipids, Oxidation and Cardiovascular Disease. In: Holtzman, J.L., Ed., Atherosclerosis and Oxidant Stress, Springer, New York, 79-95.

[4] Akoh, C.C. and Min, D.B. (2008) Food Lipids: Chemistry, Nutritionand Biotechnology. CRC Press/Taylor and Francis Group, Boca Raton.

[5] Min, D.B. (1998) Lipid Oxidation of Edible Oil. In: Min, D.B., Ed., Food Lipids: Chemistry, Nutrition and Biotechnology, Narcel Dekker, New York, 283-296.

[6] Reynolds, T. and Dweck, A. (1999) Aloe Vera Leaf Gel: A Review Update. Journal of Ethnopharmacology, 68, 3-37. https://doi.org/10.1016/S0378-8741(99)00085-9 
[7] Saritha, V., Anilakumar, K.R. and Khanum, F. (2010) Antioxidant and Antibacterial Activity of Aloe Vera Gel Extracts. International Journal of Pharmaceutical and Biological Archive, 1, 376-384.

[8] Bell, L.N. (2001) Stability Testing of Nutraceuticals and Functional Foods. In: Wildman, R.E.C., Ed., Handbook of Nutraceuticals and Functional Foods, CRC Press, New York, 501-516.

[9] Ader, P. (2000) Bioavailability and Metabolism of the Flavonol Quercetin in the Pig. Free Radical Biology and Medicine, 28, 1056-1067. https://doi.org/10.1016/S0891-5849(00)00195-7

[10] Barras, A., Mezzetti, A., Richard, A., Lazzaroni, S., Roux, S., Melnyk, P. and Monfilliette-Dupont, N. (2009) Formulation and Characterization of Polyphenol-Loaded Lipid Nanocapsules. International Journal of Pharmaceutics, 379, 270-277. https://doi.org/10.1016/j.ijpharm.2009.05.054

[11] Weiss, J., Decker, E.A., McClements, D.J., Kristbergsson, K., Helgason, T. and Awad, T. (2008) Solid Lipid Nanoparticles as Delivery Systems for Bioactive Food Components. Food Biophysics, 3, 146-154. https://doi.org/10.1007/s11483-008-9065-8

[12] Azimi, B., Nourpanah, P., Rabiee, M. and Arbab, S. (2014) Producing Gelatin Nanoparticles as Delivery System for Bovine Serum Albumin. Iranian Biomedical Journal, 18, 34-40.

[13] Freiberg, S. and Zhu, X. (2004) Polymer Microspheres for Controlled Drug Release. International Journal of Pharmaceutics, 282, 1-18. https://doi.org/10.1016/j.ijpharm.2004.04.013

[14] Nallamuthu, I., Parthasarathi, A. and Khanum, F. (2013) Thymoquinone-Loaded PLGA Nanoparticles: Antioxidant and Anti-Microbial Properties. International Current Pharmaceutical Journal, 2, 7017. https://doi.org/10.3329/icpj.v2i12.17017

[15] Zigoneanu, I.G., Astete, C.E. and Sabliov, C.M. (2008) Nanoparticles with Entrapped $\alpha$-Tocopherol: Synthesis, Characterization and Controlled Release. Nanotechnology, 19, Article ID: 105606. https://doi.org/10.1088/0957-4484/19/10/105606

[16] Qi, L., Xu, Z., Jiang, X., Hu, C. and Zou, X. (2004) Preparation and Antibacterial Activity of Chitosan Nanoparticles. Carbohydrate Research, 339, 2693-2700. https://doi.org/10.1016/j.carres.2004.09.007

[17] Dinarvand, R., Jafarzadeh Kashi, T., Eskandarion, S., Esfandyari-Manesh, M., Samadi, N., Atyabi, F. and Eshraghi, S. (2012) Improved Drug Loading and Antibacterial Activity of Minocycline-Loaded PLGA Nanoparticles Prepared by Solid/Oil/Water Ion Pairing Method. International Journal of Nanomedicine, 7, 221-234. https://doi.org/10.2147/IJN.S27709

[18] Hill, L.E., Taylor, T.M. and Gomes, C. (2013) Antimicrobial Efficacy of Poly (DL-Lactide-Co-Glycolide) (PLGA) Nanoparticles with Entrapped Cinnamon Bark Extract against Listeria Monocytogenes and Salmonella Typhimurium. Journal of Food Science, 78, N626-N632.

[19] Peng, S., Hung, W.-L., Peng, Y.-S. and Chu, I.-M. (2014) Oligoalanine-Modified PluronicF127 Nanocarriers for the Delivery of Curcumin with Enhanced Entrapment Efficiency. Journal of Biomaterials Science, Polymer Editionience, 25, 1225-1239. https://doi.org/10.1080/09205063.2014.924059

[20] Langer, R. (1990) New Methods of Drug Delivery. Science, 249, 1527-1533. https://doi.org/10.1126/science.2218494

[21] Kassama, L., Kuponiyi, A. and Kukhtareva, T. (2015) Rapid Laser-Assisted Nanosizing 
Noble Silver Nanoparticles in Plant Extracts and Physiochemical Characterization. Journal of Contemporary Research, 5, 8-18.

[22] Braca, A., De Tommasi, N., Di Bari, L., Pizza, C., Politi, M. and Morelli, I. (2001) Antioxidant Principles from Bauhinia Tarapotensis. Journal of Natural Products, 64, 892-895. https://doi.org/10.1021/np0100845

[23] Ahsan, F. (2002) Targeting to Macrophages: Role of Physicochemical Properties of Particulate Carriers-Liposomes and Microspheres-On the Phagocytosis by Macrophages. Journal of Controlled Release, 79, 29-40. https://doi.org/10.1016/S0168-3659(01)00549-1

[24] Mainardes, R.M. and Evangelista, R.C. (2005) PLGA Nanoparticles Containing Praziquantel: Effect of Formulation Variables on Size Distribution. International Journal of Pharmaceutics, 290, 137-144. https://doi.org/10.1016/j.ijpharm.2004.11.027

[25] Bhattacharyya, S.S., Paul, S. and Khuda-Bukhsh, A.R. (2010) Encapsulated Plant Extract (Gelsemium sempervirens) Poly (Lactide-Co-Glycolide) Nanoparticles Enhance Cellular Uptake and Increase Bioactivity in vitro. Experimental Biology and Medicine, 235, 678-688. https://doi.org/10.1258/ebm.2010.009338

[26] Pereira, M.C., Hill, L.E., Zambiazi, R.C., Mertens-Talcott, S., Talcott, S. and Gomes, C.L. (2015) Nanoencapsulation of Hydrophobic Phytochemicals Using Poly (Dl-Lactide-Co-Glycolide) (PLGA) for Antioxidant and Antimicrobial Delivery Applications: Guabiroba Fruit (Campomanesia xanthocarpa O. Berg) Study. LWTFood Science and Technology, 63, 100-107. https://doi.org/10.1016/j.lwt.2015.03.062

[27] Pool, H., Quintanar, D., Figueroa, J.D., Marinho Mano, C., Bechara, J.E., Godínez, L.A. and Mendoza, S. (2012) Antioxidant Effects of Quercetin and Catechin Encapsulated into PLGA Nanoparticles. Journal of Nanomaterials, 2012, 1-12. https://doi.org/10.1155/2012/145380

[28] Alam, S., Mustafa, G., Khan, Z I., Islam, F., Bhatnagar, A., Ahmad, F. and Kumar, M. (2012) Development and Evaluation of Thymoquinone-Encapsulated Chitosan Nanoparticles for Nose-to-Brain Targeting: A Pharmacoscintigraphic Study. International Journal of Nanomedicine, 7, 5705-5718. https://doi.org/10.2147/IJN.S35329

[29] Odeh, F., Ismail, S.I., Abu-Dahab, R., Mahmoud, I.S. and Al Bawab, A. (2012) Thymoquinone in Liposomes: A Study of Loading Efficiency and Biological Activity towards Breast Cancer. Drug Delivery, 19, 371-377. https://doi.org/10.3109/10717544.2012.727500

[30] Panyam, J. and Labhasetwar, V. (2003) Biodegradable Nanoparticles for Drug and Gene Delivery to Cells and Tissue. Advanced Drug Delivery Reviews, 55, 329-347. https://doi.org/10.1016/S0169-409X(02)00228-4

[31] Panyam, J., Williams, D., Dash, A., Leslie-Pelecky, D. and Labhasetwar, V. (2004) Solid-State Solubility Influences Encapsulation and Release of Hydrophobic Drugs from PLGA/PLA Nanoparticles. Journal of Pharmaceutical Sciences, 93, 1804-1814. https://doi.org/10.1002/jps.20094

[32] Wischke, C. and Schwendeman, S.P. (2008) Principles of Encapsulating Hydrophobic Drugs in PLA/PLGA Microparticles. International Journal of Pharmaceutics, 364, 298-327. https://doi.org/10.1016/j.ijpharm.2008.04.042

[33] Blanco, M. and Alonso, M. (1997) Development and Characterization of ProteinLoaded Poly (Lactideco-Glycolide) Nanospheres. European Journal of Pharmaceutics and Biopharmaceutics, 43, 287-294. https://doi.org/10.1016/S0939-6411(97)00056-8 
[34] Mathew, A., Fukuda, T., Nagaoka, Y., Hasumura, T., Morimoto, H., Yoshida, Y., Maekawa, T., Kizhikkilot Venugopal, K. and Kumar, D.S. (2012) Curcumin Loaded-PLGA Nanoparticles Conjugated with Tet-1 Peptide for Potential Use in Alzheimer's Disease. PLOS ONE, 7, e32616. http://www.plosone.org https://doi.org/10.1371/journal.pone.0032616 